\title{
Critical Role of CA1 Nicotinic Receptors on Memory Acquisition Deficit Under Induction of Total Sleep Deprivation and REM Sleep Deprivation
}

\author{
Bibi-Zahra Javad-Moosavi ${ }^{1 *}$, Gholamhassan Vaezi ${ }^{1}$, Mohammad Nasehi ${ }^{2}$, Seyed-Ali Haeri-Rouhani ${ }^{3}$, \\ Mohammad-Reza Zarrindast ${ }^{2,4-7}$ \\ 'Department of Biology, Damghan Branch, Islamic Azad University, Semnan, Iran \\ ${ }^{2}$ Cognitive and Neuroscience Research Center (CNRC), Tehran Medical Sciences Branch, Islamic Azad University, Tehran, \\ Iran \\ ${ }^{3}$ Department of Animal Biology, School of Biology, University College of Science, University of Tehran, Tehran, Iran \\ ${ }^{4}$ Medical Genomics Research Center, Tehran Medical Sciences Branch, Islamic Azad University, Tehran, Iran \\ ${ }^{5}$ Iranian National Center for Addiction Studies, Tehran University of Medical Sciences, Tehran, Iran \\ ${ }^{6}$ School of Cognitive Sciences, Institute for Research in Fundamental Sciences (IPM), Tehran, Iran \\ ${ }^{7}$ Department of Pharmacology, School of Medicine, Tehran University of Medical Sciences, Tehran, Iran
}

\begin{abstract}
Background: Sleep disorder or sleep deprivation (SD) is a common issue in today's society. Numerous evidences show that sleep is essential for proper brain performance and cognitive processes; on the other hand, cognitive functions have a broad range with learning and long-term memory as the most important ones related to attention. Since many studies show that cholinergic system has a significant role in sleep, learning, and memory, this study aims to investigate the impacts of CA1 Cholinergic Nicotinic Receptors on memory acquisition deficit which is stimulated by total sleep deprivation (TSD) and REM sleep deprivation (RSD).

Methods: In this study a water box or a multi-platform apparatus was used in order to induce TSD or RSD. In order to investigate interactions of cholinergic system and hippocampus-dependent memory, nicotinic receptor agonist (nicotine) or nicotinic receptor antagonist (mecamylamine) was injected in hippocampal CA1.

Results: According to the results of this study, 24 hours TSD or RSD decreased memory acquisition and injection of nicotine $(0.0001 \mu \mathrm{g} / \mathrm{rat})$ or mecamylamine $(0.001 \mu \mathrm{g} / \mathrm{rat})$ in TSD and RSD sham groups didn't change memory acquisition. However, injection of sub-threshold dose of nicotine $(0.0001 \mu \mathrm{g} / \mathrm{rat})$ and mecamylamine $(0.001 \mu \mathrm{g} / \mathrm{rat})$ could reduce negative effects of SD in both TSD and RSD.

Conclusion: According to the present study, cholinergic nicotinic receptors are effective in learning and memory improvement.

Keywords: Sleep; Deprivation; CA1; Nicotine; Mecamylamine
\end{abstract}

*Correspondence to Bibi Zahra Javad-Moosavi, Department of Biology, Damghan Branch, Islamic Azad University, Semnan, Iran.

Tel: +98(913)2533573.

Email:

javadmosavi60@yahoo.com

Published online March 15, 2018

Citation: Javad-Moosavi BZ, Vaezi G, Nasehi M, Haeri-Rouhani SA, Zarrindast MR. Critical role of CA1 nicotinic receptors on memory acquisition deficit Under Induction of total sleep deprivation and REM sleep deprivation. Int Clin Neurosci J. 2018;5(1):11-20. 10.15171/icnj.2018.04

\section{Introduction}

In today's world, prolonged wakefulness can be considered as a widespread phenomenon which probably occurs because of acute total sleep deprivation (TSD) or chronic partial sleep limitation. ${ }^{1}$ Birds and mammals represent two distinguished kinds of sleep including nonrapid eye movement (NREM) and rapid eye movement (REM) which can be distinguished fairly easily using electroencephalogram (EEG) and electromyogram (EMG). ${ }^{2,3}$

Studies have shown that sleep is essential to maintain energy, regulate heat, and recover tissues ${ }^{4}$; moreover, it is beneficial for proper cognitive functions. ${ }^{5,6}$ On the other hand, it seems that lack of sleep or sleep disorder will have reverse effects which may cause cognitive deficiencies such as impairment in attention, decision making, learning, and various kinds of memory. ${ }^{5,6}$ Furthermore, lack of sleep activates sympathetic system and leads to hypertension as well as increased cortisol, ${ }^{7}$ while immune responses are impaired ${ }^{8}$ and changes in mood are observed. ${ }^{9}$

Hippocampus is one of brain areas which is definitely important in most cognitive functions. ${ }^{10}$ Hippocampal formations receive multi-modal sensory data which is provided by different cortical bases, play a significant role 
in memory processes, learning, spatial coding, and also regulate emotional behaviors and anxiety. ${ }^{11}$

Numerous studies performed on humans and animals have indicated that pre-training SD leads to impairment of learning and memory coding in different types of tasks. ${ }^{6}$ In addition, it seems that hippocampus-dependent stabilization of memory is especially sensitive to sleep disorder during sleep phase immediately after learning and memory consolidation. ${ }^{11}$

In fact, studies which have investigated the impacts of sleep on learning have indicated the significance of sleep in stabilization of memory and have introduced hippocampus as a susceptible target due to outcomes associated with lack of sleep or sleep disorder. ${ }^{11}$

Existing evidence shows that SD may impair processes of neuronal formation in hippocampus and memory through reduction in $\mathrm{LTP},{ }^{12,13}$ reduction of cAMP/ PKA signaling, ${ }^{5,13}$ reduction of glutamate receptors' performance and expression. ${ }^{14,15}$. Decreasing transcription of CREB intermediary gene, and finally neurotropic gene. $^{16}$

Different stages of sleep and wakefulness are dependent on specific types of neurotransmitters and their specialized receptors such as acetyl choline, serotonin, glutamate, and so on like other animal functions. ${ }^{17,18}$

Acetyl choline is secreted by all neurons in CNS and is also found in basal forebrain. This structure sends its branches into hippocampus, neuro-cortex, and polymidbrain cholinergic complex after which it is divided and its branches go to posterior thalamus and anterior brain. ${ }^{17,19}$ It seems that this system has a significant role in sleep, wakefulness, learning and memory. ${ }^{20}$

Acetyl choline is produced in cholinergic neuron terminals by choline acetyl transferase enzyme (chAT) and is hydrolyzed by acetyl choline esterase in synaptic cleft after it has established its effects. ${ }^{21}$

Traditionally, acetyl choline receptors are divided into two main groups of muscarinic cholinergic receptors and nicotinic cholinergic receptors based on pharmacologic features. Both muscarinic and nicotinic receptors are found in great amounts in the brain. Acetyl choline nicotinic receptors are members of a superfamily of ligand gate ionic channels. ${ }^{22,23}$ Cholinergic nicotinic receptors in the brain are mostly located on glutamate secreted axon terminals as presynaptic and facilitate release of this transmitter. ${ }^{24}$ However, some are post synaptic. Muscarinic cholinergic receptors are completely different from nicotinic receptors. Large amounts of five different types of muscarinic cholinergic receptors are expressed in brain five separate simulation genes and most of them act through protein G-coupled receptors. ${ }^{21}$ Posterior hippocampus or CA1 which has a significant role in learning and memory includes high amounts of cholinergic receptors. ${ }^{25}$

In the present study, both TSDs and RSDs are expected to reduce memory acquisition, activate nAchRs of hippocampus CA1 area, provide suitable conditions for neuronal plasticizing and LTP production, and improve memory acquisition, and since the concentration of Ach increases during the RAM sleep and decreases during NREM sleep, ${ }^{20}$ and given the fact that there is RSD in the TSD as well, it is also possible that the 2 groups respond similarly to the activation of nAchRs.

It is also expected that the blockage of nAchRs by mecamylamine, followed by $\mathrm{SD}$, will reduce memory acquisition.

Since people's quality of life may change due to the effects of lack of sleep, and given that some questions are still unanswered regarding sleep function and the effects due to lack of sleep, and also considering the close relationship between sleep and wakefulness as well as cholinergic system and cognitive performance, this study aims to examine the role which is played by posterior hippocampus (CA1) cholinergic nicotinic receptors in amnesia due to RSD and TSD.

\section{Methods and Materials}

Animals

Male Wistar rats with a weight range of 220-250 g were provided from Institute for Cognitive Science Studies (ICSS) and were then used in the experiments of the present study. Plexiglas cages were used to keep animals in groups of four members under standard temperature $(22 \pm 2)$ and light/dark cycle (12/12 hours), while they could access water and food (except some short stages of the experiment) freely. Each group included 7 male rats and each rat was used only once. Application of all behavioral tests was done during light phase of light/ dark cycle. All ethical issues were considered during experiments.

\section{Stereotactic Surgery}

Anesthesia was performed by intra-peritoneal injection of ketamine hydrochloride $(50 \mathrm{mg} / \mathrm{kg}$ ) and xylazine $(5 \mathrm{mg} / \mathrm{kg})$ after which they were located in stereotaxic apparatus (Stoelting Company, IL, USA). First of all, head hair was removed, then incision of the skin was done and the surface of the skull was cleaned. In the following step, 22-guage guide cannula (with a diameter of $0.7 \mathrm{~mm}$ ) was placed one millimeter above the desired location of injection (bilaterally) based on Paxinos and Watson Atlas. ${ }^{26}$ Stereotaxic coordinates for hippocampus CA1 areas included AP: $-2 \mathrm{~mm}$ from bregma, L: \pm 1.6 sagital suture, and V: $-1.5 \mathrm{~mm}$ from the surface of the skull. Dental acrylic helped to fix cannulas. Insertion of stainless steel stylets (27-gauge) was done into the guide cannulas in order to prevent contamination. Approximately 5-7 days were required for animals' recovery after the operation. ${ }^{27,28}$

\section{Sleep Deprivation Apparatus}

Total Sleep Deprivation

In order to deprive animals from NREM sleep 
(BorjSanatAzma Co. Tehran, Iran), modified automatic TSD apparatus (water box) was used. ${ }^{29}$

This apparatus consists of a water tank made of clear Plexiglas $(120 \times 30 \times 50 \mathrm{~cm})$ divided into 4 equal boxes $(30 \times 30 \times 50 \mathrm{~cm})$, with water temperature being set at 30 centigrade degrees. Four rats were put together in the tank (one rat in each box) with the aim to keep social stability. Two small platforms (with diameter of $15 \mathrm{~cm}$ ) with a $3-\mathrm{mm}$ deep edge were located next to each other in the middle of the tank, while some holes were prepared at the surface of each platform (with diameter of $2 \mathrm{~mm}$ ) so that water could discharge easily through upward movements which protected rats against slipping or getting wet. Platform movements were done independently and automatically. In the start position, both platforms were submerged in water surface slightly. Then each platform moved upward and downward to force rats move without stopping so that they could evade from being in touch with water. The speed of movement was set at $1 \mathrm{~m} / \mathrm{s}$. Completion of each platform rotation required 30 seconds. Every platform was in its peak height $(10 \mathrm{~cm})$ over water surface for 10 seconds (in order to get water and food) (holding time) through this period. After this period of time, the platform was shifted $60 \mathrm{~mm}$ down for 2.5 seconds and was instantly raised for 2.5 seconds to the primary position. The day before application of various sleep deprivation (SD) protocols, the rats were familiarized with the water box, so that their stress level was reduced for 30 minutes. As a result, the rats learned remaining at the junction of two platforms and going from the sinking platform to the other one in a short movement. The rats could access water and food which was always provided above the box freely over this period. Behavioral observations obtained during daily SD indicated that rats were awaked $100 \%$ of the time when platform technique was applied. ${ }^{29}$

The rats were deprived from sleep for 24 hours (TSD) for the purposes of the present study. Standard conditions were provided to keep animals (12:12 hours light-dark cycle in a temperature under control $\left[22 \pm 2^{\circ} \mathrm{C}\right]$ ) during SD phase. Potential effects of stress because of new environment were evaluated using control (Sham) groups in similar situations when the apparatus was off.

\section{REM Sleep Deprivation}

Multiple-platform apparatus (BorjSanatAzma Co, Tehran, Iran) was used for RSD. To apply this method rats should be located in a water tank $(90 \times 50 \times 50 \mathrm{~cm})$ consisting of some circular platforms with diagonals of $7 \mathrm{~cm}$. In the present study each platform was placed $2 \mathrm{~cm}$ above water level. During experiments the rats were free to move through the tank and go from one platform to another. In the above mentioned technique when the rats went into REM sleep their muscles were relaxed and they fell into water which consequently led to their wakefulness. Thus, the animals were deprived from REM sleep all the time period they spent in water tank. On the other hand, platforms with a diagonal of $15 \mathrm{~cm}$ were applied for sham of RSD animals, so that the animals could have REM sleep conveniently. Standard water temperature was monitored during this experiment, while light-dark cycle (12/12) was also considered and food and water were likewise provided for the animals. ${ }^{30}$ The rats were deprived from REM sleep for 24 hours (RSD) in this study.

\section{Intra-CA1 Injection}

Removal of stainless steel stylets from the guide cannula and replacement with 27-gauge injection needles $(1 \mathrm{~mm}$ lower than the tip of the guide cannula) was performed so that drugs could be injected. Drug injections were done manually in total volume of $1 \mu \mathrm{L} /$ rat $(0.5 \mu \mathrm{L}$ per each cannula) during a period of 60 seconds and using Hamilton syringe $(2 \mu \mathrm{L})$ while needles were put in the place for 60 seconds so that drug injection could be facilitated. After returning the stylets the animals were transferred into their cages. ${ }^{29}$

\section{Step-Through Apparatus for Memory Assessment} Passive avoidance learning apparatus included a box divided into 2 partitions with equivalent sizes $(20 \times 20 \times 30)$ by a wall. A guillotine door was located in the middle of the wall with dimensions of $7 \times 9(\mathrm{~cm})$ and could be removed when it was required. One part of the apparatus was designed with white Plexiglas walls and floor, while a $25 \mathrm{~W}$ electric lamp $50 \mathrm{~cm}$ above the apparatus floor lit this section. The other part was set to be dark with black Plexiglas walls, while steel rods with $1 \mathrm{~cm}$ intervals from each other were located at the floor. These rods were connected to a stimulator with a connection wire so that foot shocks could be produced during the experiment. An insulated stimulator was used to deliver periodic electric shocks (50 Hz, 3 seconds, $1 \mathrm{~mA}$ intensity) to the grid floor of the dark compartment. ${ }^{31}$

\section{Behavioral Procedures \\ Training}

At least 30 minutes before the experiments, animals were moved into the test room. After this step each animal was located mildly in the light part of the apparatus. Then the guillotine door was opened after five seconds so that the animal could enter into the dark part of the apparatus. The delay which every animal showed to enter into the dark compartment was recorded in this stage. Exclusion was based on the delay time and those animals which had been waited more than 120 seconds to enter into the dark compartment were excluded from the experiments. When 4 paws of the animal were totally entered into the next compartment, the guillotine door was closed and the rat returned to its cage after 10 seconds (habituation trial). Application of learning trial was done 30 minutes after habituation trial. Five seconds after placement of the animal in the light compartment, the guillotine door was opened. Immediately after the animal entered into the 
dark compartment, the door was closed and shock (50 $\mathrm{Hz}, 1 \mathrm{~mA}, 3$ seconds) was instantly transferred to animal feet through the floor of the dark compartment. After 20 seconds, removal of the rat from the apparatus was performed and the animal was accordingly delivered to its cage temporarily. Procedure repetition took place after two minutes. Each time the rat entered totally into the dark compartment, electric shock was delivered to its feet. The end of learning trial was the time in which the rat did not leave the light compartment and stayed there for 120 consecutive seconds. The number of trials (entrance into the dark chamber) was recorded and accordingly showed that all animals learned with at most 3 trials. This section was performed 5 minutes after drug injection. ${ }^{27}$

\section{Retrieval Test}

Retrieval test was implemented 24 hours after training was finished so that long-term memory could be identified. On the test day electric shock wan not administered. Each animal was located in the light compartment for twenty seconds after which the door was opened and delay in entrance into the dark compartment was recorded during the period which lasted for 300 seconds. ${ }^{29}$

\section{Pain Test}

Hot plate apparatus (BorjSanatAzma Co, Tehran, Iran) was used in order to evaluate changes in pain threshold. This apparatus includes a rectangular cast-iron plate $(20 \times 25)$ equipped with a thermostat, power supply $(220 \mathrm{~W})$, and a holding cylinder (with a diagonal of 20 and height of 30 $\mathrm{cm}$ ). Temperature of apparatus was set at $50 \pm 1$ centigrade degree in hot plate tests, while reaction time to thermal pain was recorded as licking paws or special changes in rats' steps. Cut-off time of the test was 60 seconds. This test was performed for each rat after memory training. Results were expressed according to delay time to react to pain (pain latency) and based on seconds. ${ }^{32}$

\section{Locomotor Activity Evaluation}

Animals' locomotion apparatus (BorjSanatAzma Co, Tehran, Iran) consisted of a transparent Perspex container (with a height of $40 \times 30 \times 30 \mathrm{~cm}$ ). This apparatus included a gray Perspex panel (with a thickness of $2.2 \times 30 \times 30 \mathrm{~cm}$ ) with 16 photocells which separated the container into 16 squares with equal sizes. Locomotor activities were evaluated as the number of movements from one square to another during 300 seconds. This test was done at trail day. ${ }^{33}$

\section{Drugs}

Drugs that were used in this study had been supplied from Tocris (Tocris Bioscience, United Kingdom) and included nicotine (nicotinic receptor agonist) and mecamylamine (nicotinic receptor antagonist). Preparation of drugs was done immediately before application of injections at doses of $0.0001,0.001,0.01$ and $0.1 \mu \mathrm{g} /$ rat.

\section{Statistical Analysis}

The results were evaluated statistically considering normal distribution of data and also using independent $t$ test or one-way analysis of variance (ANOVA). Following significant $\mathrm{F}$ value, more analyses were done for pairedgroup comparisons with the help of post hoc Tukey tests. All data are expressed as mean \pm standard error of the mean (SEM). In all comparisons, $P<0.05$ represented statistical significance.

\section{Verification of Cannula Supplies}

Each rat was anesthetized after the above mentioned test sessions, and $0.05 \mu \mathrm{L}$ solution of $4 \%$ methylene-blue was injected into CA1 in order to confirm the site of cannulation. Next the rat's head was separated after which removal of the brain and its fixation in formaldehyde (10\%) for one week was performed. After this period of time, the brain was cut and the sites of injections were verified according to Paxinos and Watson. ${ }^{26}$

\section{Experimental Design}

Experiment1: Effects of Pre-train Intra-CA1 Injection of Nicotine or Mecamylamine on Memory Acquisition, Pain and Locomotor Activities

Eight groups of animals have been allocated to the present trial. Saline $(1 \mu \mathrm{L} / \mathrm{rat})$, various doses of nicotine $(0.0001$, $0.001,0.01$ and $0.1 \mu \mathrm{g} / \mathrm{rat})$ or mecamylamine $(0.001,0.01$ and $0.1 \mu \mathrm{g} / \mathrm{rat}$ ) were applied on the rats. Pre-train drug injection was used for all cases. This experiment aimed to characterize sub-threshold and effective doses of each drug in the normal conditions.

Experiment2: The Impact of TSD on Memory Acquisition, Pain and Locomotor Activities in Presence and Absence of Nicotine or Mecamylamine

Six groups of animals have been allocated to the present trial. Intra-CA1 injection of saline $(1 \mu \mathrm{L} / \mathrm{rat})$, nicotine $(0.0001 \mu \mathrm{g} / \mathrm{rat})$ or mecamylamine $(0.001 \mu \mathrm{g} / \mathrm{rat})$, was performed for the animals 5 minutes before memory training in the sham of TSD or TSD. This experiment aimed to identify if sub-threshold dose of drugs in the sham condition could improve the reactions which were induced by TSD.

Experiment3: The Impact of RSD on Memory Acquisition, Pain and Locomotor Activities in Presence and Absence of Nicotine or Mecamylamine

Six groups of animals have been allocated to this experiment. The animals received intra-CA1 injection of saline $(1 \mu \mathrm{L} / \mathrm{rat})$, nicotine $(0.0001 \mu \mathrm{g} / \mathrm{rat})$ or mecamylamine $(0.001 \mu \mathrm{g} / \mathrm{rat}), 5$ minutes before memory training in the sham of RSD or RSD. This experiment aimed to identify if sub-threshold dose of drugs in the sham condition could improve the reactions which were induced by RSD. 


\section{Results}

The Impact of Pre-train Intra-CA1 Injection of Nicotine and Mecamylamine on Memory, Pain and Locomotor Activities

One-way ANOVA and post hoc Tukey test showed that intra-CA1 injection of nicotine in the normal rats at doses 0.001 and 0.1 but not 0.0001 and $0.01 \mu \mathrm{g} /$ rat reduced memory acquisition $[\mathrm{F}(3,24)=22.681 ; P<0.05$; Figure 1 , panel $1 \mathrm{~A}]$, but it did not alter pain response $[\mathrm{F}(3$, $24)=4.698, P>0.05$; Figure 1 , pane1 $2 \mathrm{~A}$ ] while nicotine at doses 0.01 and 0.1 but not 0.0001 and $0.001 \mu \mathrm{g} / \mathrm{rat}$ increased response induced by loco-motor activity in normal rats $[\mathrm{F}(3,24)=49.40, P<0.05$; Figure 1, panel 3A].

One-way ANOVA and post hoc Tukey test showed that intra-CA1 injection of mecamylamine in the normal rats at all doses reduced memory acquisition $[\mathrm{F}(3,24)$ =6.252; $\mathrm{P}<0.05$; Figure 1, panel 1B], but it did not alter pain response $[\mathrm{F}(3,24)=6.592, P>0.05$; Figure 1 , pane1 2B] while mecamylamine at all doses increased response induced by loco-motor activity $[\mathrm{F}(3,24)=24.69, P<0.05$; Figure 1, panel 3B].

In according of these results we selected nicotine at dose of $0.0001 \mu \mathrm{g} / \mathrm{rat}$ and mecamylamine at dose of $0.001 \mu \mathrm{g} / \mathrm{rat}$ as sub-threshold dose of these drugs in next experiments.

The Effects of TSD on Memory Acquisition, Pain and Locomotor Activities in Presence and Absence of Nicotine and Mecamylamine

One-way ANOVA and post hoc Tukey analysis indicated that sub-threshold dose of nicotine and mecamylamine (that selected according to previous experiment) did not alter memory acquisition $[\mathrm{F}(2,18)=2.930 ; P>0.05$; Figure 2, panel 1A], while mecamylamine but not nicotine increased time interval required for reaction to pain $[\mathrm{F}$ $(2,18)=27.653 ; P<0.05$; Figure 2, panel 2A]. However, nicotine but not mecamylamine decreased loco-motor activity $[\mathrm{F}(2,18)=12.542 ; P<0.05$; Figure 2, panel $3 \mathrm{~A}]$.

The $t$ test analysis showed that TSD could significantly decrease memory acquisition $(\mathrm{t}=28 ; P<0.05$; Figure 2, panel 1).and loco-motor activity $(\mathrm{t}=3.374, P<0.05$; Figure 2 , panel 3) while it increased time interval required for reaction to pain ( $t=4.95 ; P<0.05$; Figure 2 , panel 2$)$.

One-Way ANOVA and post hoc Tukey test indicated that nicotine and mecamylamine restitute the amnesia which has been brought about by TSD $[\mathrm{F}(2,18)=12.888$, $P<0.05$; Figure 2, panel $1 \mathrm{~B}$ ], while both drugs decreased

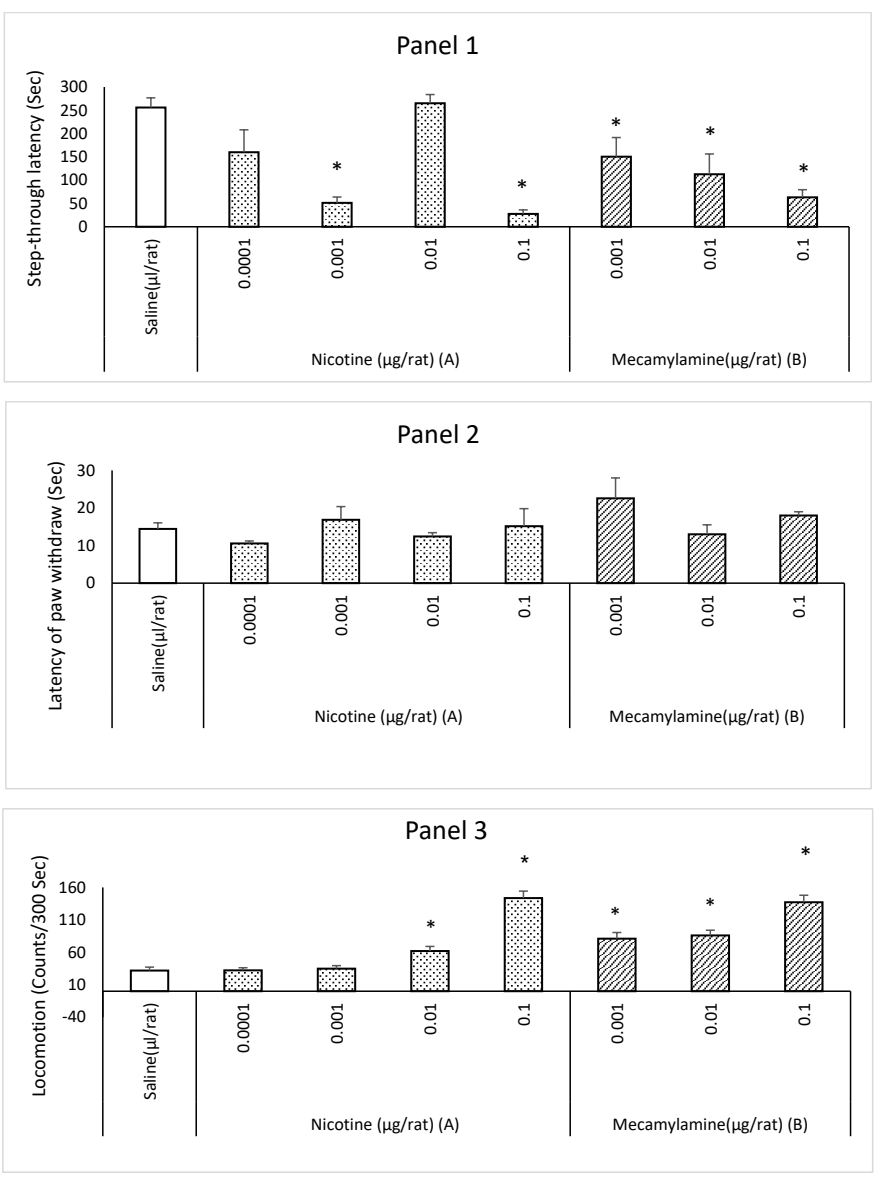

Figure 1. The Effect of Pre-training Intra-CA1 Injection of Nicotine (A) or Mecamylamine (B) on Memory Acquisition (Panel A), Latency to Paw Withdraw (Panel B) and Locomotion (Panel C). The data have been shown as Mean \pm SEM for seven rats for each group. $* P<0.05$, $* * P<0.01$ and $* * * P<0.001$ as compared to saline control group. 


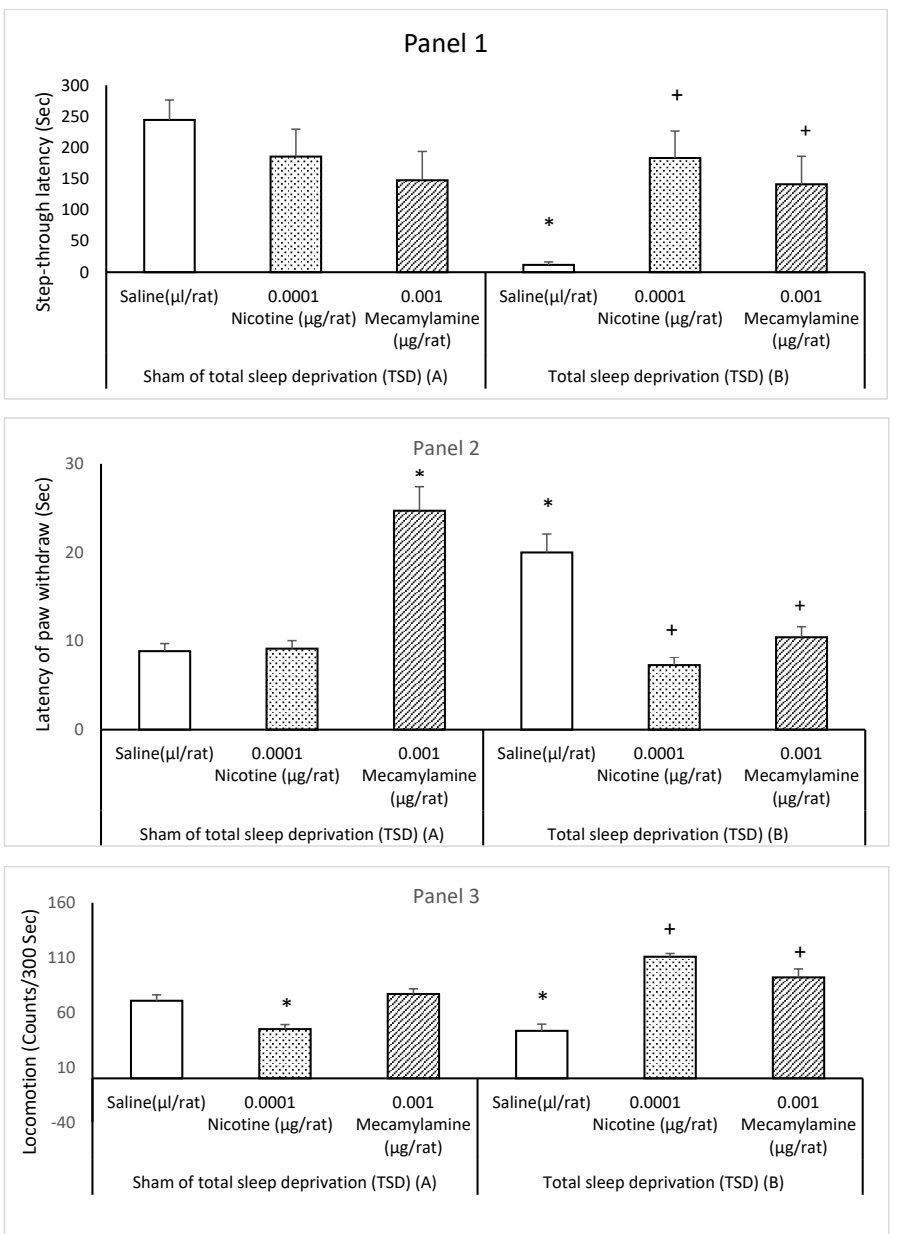

Figure 2. The Impact of Pre-training Intra-CA1 Injection of Nicotine or Mecamylamine on Memory Acquisition (Panel 1), Latency to Paw Withdraw (Panel 2) and Locomotor Activity (Panel 3) on Sham of TSD (A) or TSD Rats (B). The data have been shown as Mean \pm SEM for seven rats per group. ${ }^{*} P<0.05,{ }^{* *} P<0.01$ and ${ }^{* * *} P<0.001$ as compared to saline/sham of TSD control group. ${ }^{+} P<0.05,{ }^{++} P<0.01$ and ${ }^{+++} P<0.001$ as compared to saline/TSD control group.

time interval required for reaction to pain $[\mathrm{F}(2$, $18)=20.084, P<0.05$; Figure 2, panel $2 \mathrm{~B}]$ and increased loco-motor activity $[\mathrm{F}(2,18)=34.655 ; P<0.05$; Figure 2, panel 3B]

3-3: The Impact of RSD on Memory Acquisition, Pain and Locomotor Activities in Presence and Absence of Nicotine and Mecamylamine

One-way ANOVA and post hoc Tukey analysis indicated that sub-threshold dose of nicotine and mecamylamine (that selected according to experiment 1 ) did not alter memory acquisition $[\mathrm{F}(2,18)=0.139 ; P>0.05$; Figure 3 , panel $1 \mathrm{~A}$ ] in the sham RSD rats. However, mecamylamine but not nicotine increased hot plate latency $[\mathrm{F}(2,18)$ $=15.008$. $P<0.05$; Figure 3, panel 1B]. and loco-motor activity $[\mathrm{F}(2,18)=4.933 ; P<0.05$; Figure 3 , panel $1 \mathrm{C}]$.

The $t$ test analysis showed that RSD could significantly decrease memory acquisition $(t=5.348, P<0.05$; Figure 3, panel 1$)$ and loco-motor activity $(t=7.50, P<0.05$; Figure 3 , panel 3) but it did not alter time interval required for reaction to pain $(t=12.50, P>0.05$; Figure 3 , panel 2$)$ compared to sham control group.

One-way ANOVA and post hoc Tukey test showed that nicotine and mecamylamine restored amnesia induced by $\operatorname{RSD}[\mathrm{F}(2,18)=14.618 ; P<0.05$; Figure 3, panel $1 \mathrm{~B}]$ but both of drugs did not alter time interval required for reaction to pain $[\mathrm{F}(2,18)=5.215, P>0.05$; Figure 3 , panel $2 \mathrm{~B}$, while mecamylamine but not nicotine increased loco-motor activity in $\mathrm{RSD}$ rats $[\mathrm{F}(2,18)=8.373, P<0.05$; Figure 3, panel 3B].

\section{Discussion}

Investigating the Impact of Nicotine and Mecamylamine on Behaviors in Normal Animals The present study showed that some doses of nicotine caused memory acquisition reduction in normal rats, while some other doses of this drug did not affect memory; on the other hand, all doses of mecamylamine led to memory reduction. Moreover, the study also showed no significant difference in TSD and RSD sham groups regarding the effects of different doses of drugs on memory acquisition. 


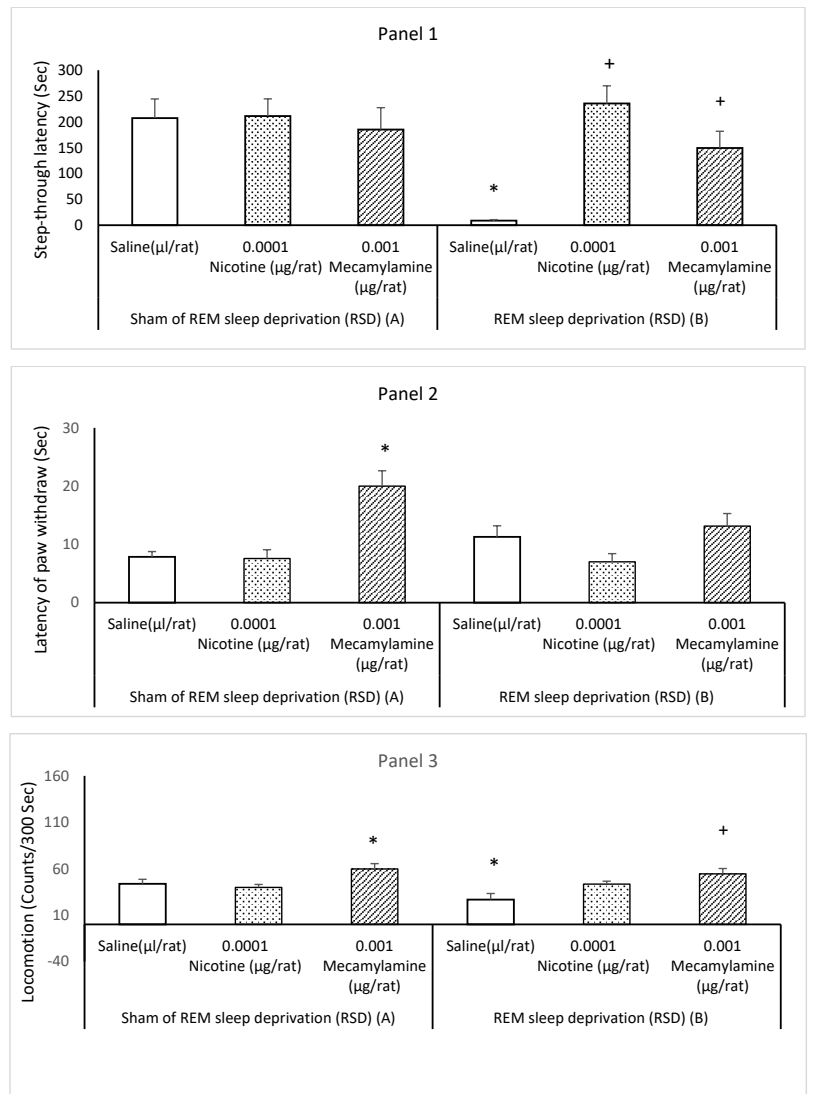

Figure 3. The Impact of Pre-training intra-CA1 Injection of Nicotine or Mecamylanine on memory acquisition (panel 1), latency to paw withdraw (Panel 2) and Locomotor Activity (Panel 3) on Sham of RSD (REM sleep deprivation; A) or RSD rats (B). The data have been shown as Mean \pm SEM for seven rats per group. ${ }^{*} P<0.05,{ }^{* *} P<0.01$ and ${ }^{* * *} P<0.001$ as compared to saline/sham of RSD control group. ${ }^{+} P<0.05$, ${ }^{++} P<0.01$ and ${ }^{+++} P<0.001$ as compared to saline/RSD control group.

Some studies have referred to the role of acetyl choline ${ }^{34}$ in learning and memory through its importance in neuronal plasticity and have introduced it as a key neuro-transmitter (which produces the driving force of neuronal activity to establish learning and memory in hippocampal system). ${ }^{35,36}$ Muscarinic and nicotinic cholinergic receptors have modulatory effects on acetyl choline performance. ${ }^{37}$ Different experimental studies on humans and animals have led to contradictory results regarding the effects of nicotine which is a nicotinic acetyl choline specific stimulus. Although some researchers have reported nicotine-induced memory improvement, some others have not observed any effects or have conversely reported its negative effects. ${ }^{24}$

Some studies show that stimulation of acetyl choline nicotinic receptor with one application of nicotine in normal rats can improve short-term memory, while it doesn't affect long-term memory. ${ }^{24}$

Other studies state that there is a reverse U-shaped response dose for cholinergic system activity. This pattern suggests that Ach has an optimal range for successful activity in hippocampus. ${ }^{38-40}$ Although this pattern does not describe different behavioral responses sufficiently, molecular and neurochemical effects of nicotine suggest a particular molecular framework leading to different behaviors.

It seems that the primary aim of nicotine in brain includes depolarization and stimulation of nAchRs, while its secondary effects include releasing glutamate and GABA inside a tissue. Therefore, according to another theory which can justify different effects of nicotine, these various functions are a consequence of different activities by nicotinic and non-nicotinic receptors. Different antagonists of acetyl choline nicotinic receptor may apply their various effects on memory performance through selective function of these compounds in subtypes of nicotinic receptors or non-nicotinic receptors. ${ }^{24}$

According to some findings, mecamylamine can have different separate effects on learning and memory processes. ${ }^{41}$ Some findings suggest that application of mecamylamine in some doses may lead to impairment of cognitive functions through disruption of anterior striatal BDNF signaling. ${ }^{42,43}$

In this study, mecamylamine is also expected to reduce memory by using different methods such as reducing BDNF signaling, blocking nAchRs, decreasing the concentration of Ach and decreasing the plasticity of the neuron. 
The Impact of TSD and RSD on Memory Acquisition, Pain and Locomotor Activity

SD affects different phases of memory formation. ${ }^{6}$ Findings which have been acquired from research done on human and animals indicate that even a slight SD or mild sleep disorder will weaken the establishment of hippocampus-dependent new memory. Although many studies show that sleep disorder will be able to affect hippocampus-dependent memory before learning, it is not obvious if this deficiency is due to impairment of memory coding or weak memory consolidation after it. ${ }^{11}$

In the present study both TSD and RSD led to reduction of memory acquisition. In line with our results, Tadavarty et al found that SD increases LTD in hippocampus CA1 area significantly. ${ }^{44}$ Given the fact that increased LTD can lead to consistent reduction in learning and memory consolidation, ${ }^{45}$ researchers suggest that increased LTD may be associated with cognitive deficiencies observed after SD. ${ }^{44}$ Consistent with these findings, Kopp et al showed that SD weakens LTP, while it induces LTD. ${ }^{46}$

Studies have also shown that REM sleep SD leads to significant deficit in maintaining hippocampal LTP, while NREM sleep disorders do not represent any deficits in LTP. ${ }^{12}$ It has been also proposed that adenosine which is one of neuronal modulators in brain may possibly have a role in learning and memory impairment induced by $\mathrm{SD}^{47,48}$ Adenosine is observed because of damage in ATP and convolute adenosine monophosphate ${ }^{25,48}$ whose concentration goes up in the period of wakefulness and goes down while asleep. ${ }^{49,50}$ Florian suggests that ATP derived from Astrocytes as well as increased adenosine during SD affect hippocampus synaptic plasticity and hippocampus-dependent memory. ${ }^{51} \mathrm{SD}$ can also prevent cAMP-PKA signaling in hippocampus and accordingly interrupts some forms of LTP in hippocampus. ${ }^{13}$

The cAMP signaling suppression due to SD can influence neuronal plasticity and memory operations through different procedures. Weak cAMP-PKA signaling may have a direct influence on phosphorylation and expression of glutamate receptors subtypes existing in cellular membrane or can lead to reduction of phosphorylation and activity of CREB protein transcription agent which regulates expression of a wide range of genes associated to synaptic plasticity, while the level of protein and BDNF mRNA which is an important neurogenesis promoter will decrease. ${ }^{52,53}$

Another important component which may cause cognitive disorders following SD is reduction of expression and function of NMDA receptors which can play a significant role in all three memory stages including learning, consolidation, and retrieval..$^{54}$ For example, while genetic improvement of performance in NMDA receptor leads to memory improvement in mature rats, ${ }^{55}$ receptors' blockage leads to learning and memory disorder. ${ }^{56}$

In addition to changes in performance and expression of glutamate receptors, SD can also affect the amount of glutamate release, ${ }^{56}$ so that high amounts of glutamate due to long-term wakefulness will lower AMPA and NMDA receptors expression which will subsequently lead to unfavorable performance and eventually neuronal plasticity disorder..$^{58}$ In the present study, TSD and RSD led to a significant decrease in memory which was consistent with other studies and according to our expectation.

The Impact of Nicotine and Mecamylamine on Behaviors-Induced by RSD and TSD

$\mathrm{BF}$ includes subcortical structures rich in cholinergic neurons and can play a significant role in activation of cortex; moreover, its damage in many neurodegenerative diseases such as Alzheimer leads to multiple problems in attention, learning, and memory. ${ }^{20}$ Primary clinical studies have not reported changes in the number, structure, and performance of mAchRs cholinergic muscarinic receptors, while reduction of some subtypes of cholinergic nicotinic receptors has been observed in cortex and hippocampus. ${ }^{20}$

$\mathrm{BF}$ cholinergic neurons are only a small range of neuron population in this structure and other neurons include glutamatergic and GABAergic subtypes showing different firing and activation patterns during sleep and wakefulness, and cortical activity. ${ }^{20}$ Studies show that activation of $\mathrm{BF}$ cholinergic neurons through PKA/ PKC signaling pathways and their intra-hippocampus dependent proteins can lead to attention, learning, and memory. ${ }^{25}$

In this study, injection of nicotine has probably improved memory in both TSD and RSD through activation of cholinergic system.

Some studies state that nicotine increases activity of cholinergic nicotinic receptors (nAchRs), particularly inside hippocampus ${ }^{59}$ increases the level of attention, ${ }^{40,60}$ and can strengthen memory acquisition and consolidation. ${ }^{59}$ Nicotine can also lead to release of other neuro-transmitters such as serotonin, GABA, norepinephrine, and glutamate all of which are necessary for memory formation, through activation of nAchRs which are presynaptic. ${ }^{59}$

In our study, according to our expectations and prediction in both groups, TSD and RSD intervention with nicotine improved memory acquisition. It seems that activating nAchRs initially increases intracellular $\mathrm{Ca}^{2+}$ by activating many signaling pathways such as:

- Increased activity of NMDA receptors, LTP enhancement

- Enhancing PKC activation and signaling pathways of ERK1,2 and AMPA

- Enhancing PKA /Camp activation and activating ERK1 signaling path 2; and

- Increased expression of CREB gene which leads to memory improvement. ${ }^{34,61}$

Nicotine intervention can also enhance memory acquisition through effects on other neurotransmitters 
involved in memory processes. Intervention with mecamylamine improved memory acquisition in both TSD and RSD groups despite expectation.

It is proposed that most effects of mecamylamine on attention and cognitive activities can be justified by NMDA receptors blockage. ${ }^{59}$ Some findings indicate that glutamate increases during long-term wakefulness and REM sleep, while it decreases in the period of NREM sleep. ${ }^{58}$ On the other hand, we know that increased glutamate concentration can induce apoptosis. ${ }^{62}$ Therefore, during deprivation of NREM and following increased glutamate concentration, mecamylamine can decrease negative effects of increased glutamate level through NMDA receptors blockage. ${ }^{63}$ We know that activation of dopaminergic D2 receptors decreases cAMP/PKA. ${ }^{64}$

Therefore, mecamylamine, as a dopaminergic antagonistic system, increases attention and improves memory through dopaminergic receptors blockage ${ }^{59}$ and increasing cAMP levels. Treatment with mecamylamine can also modulate negative effects of SD in RSD conditions.

As it was mentioned, it is probable that mecamylamine exerts many of its effects on memory by activating or blocking other receptors in the CA1 and activating some effective signaling pathways to improve memory performance.

\section{Conflict of Interest Disclosures}

The authors declare that they have no conflict of interests.

\section{Ethical Statement}

All ethical issues were considered during experiments.

\section{References}

1. Alhola P, Polo-Kantola P. Sleep deprivation: Impact on cognitive performance. Neuropsychiatr Dis Treat. 2007;3(5):553-67.

2. Lesku JA, Roth TC 2nd, Amlaner CJ, Lima SL. A phylogenetic analysis of sleep architecture in mammals: the integration of anatomy, physiology, and ecology. Am Nat. 2006;168(4):44153. doi: $10.1086 / 506973$.

3. Lesku JA, Roth TC, Rattenborg NC, Amlaner CJ, Lima SL. Phylogenetics and the correlates of mammalian sleep: a reappraisal. Sleep Med Rev. 2008;12(3):229-44. doi: 10.1016/j. smrv.2007.10.003.

4. Maquet $\mathrm{P}$. The role of sleep in learning and memory. Science. 2001;294(5544):1048-52. doi: 10.1126/science.1062856.

5. Havekes R, Vecsey CG, Abel T. The impact of sleep deprivation on neuronal and glial signaling pathways important for memory and synaptic plasticity. Cell Signal. 2012;24(6):1251-60.

6. Abel T, Havekes R, Saletin JM, Walker MP. Sleep, plasticity and memory from molecules to whole-brain networks. Curr Biol. 2013;23(17):R774-88. doi: 10.1016/j.cub.2013.07.025.

7. Lac G, Chamoux A. Elevated salivary cortisol levels as a result of sleep deprivation in a shift worker. Occup Med (Lond). 2003;53(2):143-5.

8. Spiegel K, Knutson K, Leproult R, Tasali E, Van Cauter E. Sleep loss: a novel risk factor for insulin resistance and Type 2 diabetes. J Appl Physiol (1985). 2005;99(5):2008-19. doi: 10.1152/japplphysiol.00660.2005.

9. Philibert I. Sleep loss and performance in residents and nonphysicians: a meta-analytic examination. Sleep. 2005;28(11):1392-402.
10. Lavenex P, Banta Lavenex P, Amaral DG. Postnatal development of the primate hippocampal formation. Dev Neurosci. 2007;29(1-2):179-92. doi: 10.1159/000096222.

11. Kreutzmann JC, Havekes R, Abel T, Meerlo P. Sleep deprivation and hippocampal vulnerability: changes in neuronal plasticity, neurogenesis and cognitive function. Neuroscience. 2015;309:173-90. doi: 10.1016/j.neuroscience.2015.04.053.

12. Ishikawa $A$, Kanayama $Y$, Matsumura $H$, Tsuchimochi $H$, Ishida $Y$, Nakamura S. Selective rapid eye movement sleep deprivation impairs the maintenance of long-term potentiation in the rat hippocampus. Eur J Neurosci. 2006;24(1):243-8. doi: 10.1111/j.1460-9568.2006.04874.x.

13. Vecsey CG, Baillie GS, Jaganath D, Havekes R, Daniels A, Wimmer $\mathrm{M}$, et al. Sleep deprivation impairs cAMP signalling in the hippocampus. Nature. 2009;461(7267):1122-5. doi: 10.1038/nature08488.

14. Ravassard P, Pachoud B, Comte JC, Mejia-Perez C, ScoteBlachon C, Gay N, et al. Paradoxical (REM) sleep deprivation causes a large and rapidly reversible decrease in long-term potentiation, synaptic transmission, glutamate receptor protein levels, and ERK/MAPK activation in the dorsal hippocampus. Sleep. 2009;32(2):227-40.

15. Hagewoud R, Havekes R, Novati A, Keijser JN, Van der Zee EA, Meerlo P. Sleep deprivation impairs spatial working memory and reduces hippocampal AMPA receptor phosphorylation. J Sleep Res. 2010;19(2):280-8. doi: 10.1111/j.13652869.2009.00799.x.

16. Alhaider IA, Aleisa AM, Tran TT, Alkadhi KA. Sleep deprivation prevents stimulation-induced increases of levels of P-CREB and BDNF: protection by caffeine. Mol Cell Neurosci. 2011;46(4):742-51. doi: 10.1016/j.mcn.2011.02.006.

17. Brown RE, Basheer R, McKenna JT, Strecker RE, McCarley RW. Control of sleep and wakefulness. Physiol Rev. 2012;92(3):1087187. doi: 10.1152/physrev.00032.2011.

18. Watson CJ, Baghdoyan HA, Lydic R. Neuropharmacology of Sleep and Wakefulness: 2012 Update. Sleep Med Clin. 2012;7(3):469-86. doi: 10.1016/j.jsmc.2012.06.010.

19. Benedito MA, Camarini R. Rapid eye movement sleep deprivation induces an increase in acetylcholinesterase activity in discrete rat brain regions. Braz J Med Biol Res. 2001;34(1):103-9.

20. Irmak SO, de Lecea L. Basal forebrain cholinergic modulation of sleep transitions. Sleep. 2014;37(12):1941-51. doi: 10.5665/ sleep.4246.

21. Rand JB. Acetylcholine. WormBook: the online review of C elegans biology. 2007:1-21.

22. Doyle DA. Structural changes during ion channel gating. Trends Neurosci. 2004;27(6):298-302. doi: 10.1016/j. tins.2004.04.004.

23. Pohanka M. Alpha7 nicotinic acetylcholine receptor is a target in pharmacology and toxicology. Int J Mol Sci. 2012;13(2):221938. doi: 10.3390/ijms13022219.

24. Hefco V, Yamada K, Hefco A, Hritcu L, Tiron A, Olariu A, et al. Effects of nicotine on memory impairment induced by blockade of muscarinic, nicotinic and dopamine D2 receptors in rats. Eur J Pharmacol. 2003;474(2-3):227-32.

25. Parfitt GM, Campos RC, Barbosa AK, Koth AP, Barros DM. Participation of hippocampal cholinergic system in memory persistence for inhibitory avoidance in rats. Neurobiol Learn Mem. 2012;97(2):183-8. doi: 10.1016/j.nlm.2011.12.001.

26. Paxinos G, Watson C. The rat brain in stereotaxic coordinates. 6th ed. London, UK: Academic Press; 2007.

27. Hosseini N, Nasehi M, Radahmadi M, Zarrindast MR. Effects of CA1 glutamatergic systems upon memory impairments in cholestatic rats. Behav Brain Res. 2013;256:636-45. doi: 10.1016/j.bbr.2013.08.018.

28. Naseri $\mathrm{MH}$, Hesami-Tackallou $\mathrm{S}$, Torabi-Nami M, Zarrindast MR, Nasehi M. Involvement of the CA1 GABAA receptors in MK-801-induced anxiolytic-like effects: an isobologram 
analysis. Behav Pharmacol. 2014;25(3):197-205. doi: 10.1097/ fbp.0000000000000037.

29. Norozpour Y, Nasehi M, Sabouri-Khanghah V, Torabi-Nami M, Zarrindast MR. The effect of CA1 alpha2 adrenergic receptors on memory retention deficit induced by total sleep deprivation and the reversal of circadian rhythm in a rat model. Neurobiol Learn Mem. 2016;133:53-60. doi: 10.1016/j.nlm.2016.06.004.

30. Hajali V, Sheibani V, Ghazvini H, Ghadiri T, Valizadeh T, Saadati $\mathrm{H}$, et al. Effect of castration on the susceptibility of male rats to the sleep deprivation-induced impairment of behavioral and synaptic plasticity. Neurobiol Learn Mem. 2015;123:1408. doi: 10.1016/j.nlm.2015.05.008.

31. Ahmadi-Mahmoodabadi N, Nasehi M, Emam Ghoreishi M, Zarrindast MR. Synergistic effect between prelimbic 5-HT3 and CB1 receptors on memory consolidation deficit in adult male Sprague-Dawley rats: An isobologram analysis. Neuroscience. 2016;317:173-83. doi: 10.1016/j.neuroscience.2015.12.010.

32. Nascimento DC, Andersen ML, Hipolide DC, Nobrega JN, Tufik S. Pain hypersensitivity induced by paradoxical sleep deprivation is not due to altered binding to brain muopioid receptors. Behav Brain Res. 2007;178(2):216-20. doi: 10.1016/j.bbr.2006.12.016.

33. Nasehi M, Tabatabaie M, Khakpai F, Zarrindast MR. The effects of CA1 5HT4 receptors in MK801-induced amnesia and hyperlocomotion. Neurosci Lett. 2015;587:73-8. doi: 10.1016/j.neulet.2014.12.019.

34. GouldTJ, Wilkinson DS, Yildirim E, Poole RL, Leach PT, Simmons SJ. Nicotine shifts the temporal activation of hippocampal protein kinase $\mathrm{A}$ and extracellular signal-regulated kinase $1 / 2$ to enhance long-term, but not short-term, hippocampusdependent memory. Neurobiol Learn Mem. 2014;109:151-9. doi: 10.1016/j.nlm.2014.01.009.

35. Gold PE. Coordination of multiple memory systems. Neurobiol Learn Mem. 2004;82(3):230-42. doi: 10.1016/j. nlm.2004.07.003.

36. Hasselmo ME, McGaughy J. High acetylcholine levels set circuit dynamics for attention and encoding and low acetylcholine levels set dynamics for consolidation. Prog Brain Res. 2004;145:207-31. doi: 10.1016/s0079-6123(03)45015-2.

37. James JR, Nordberg A. Genetic and environmental aspects of the role of nicotinic receptors in neurodegenerative disorders: emphasis on Alzheimer's disease and Parkinson's disease. Behav Genet. 1995;25(2):149-59.

38. Hasselmo ME. The role of acetylcholine in learning and memory. Curr Opin Neurobiol. 2006;16(6):710-5. doi: 10.1016/j.conb.2006.09.002.

39. Dumas JA, Newhouse PA. The cholinergic hypothesis of cognitive aging revisited again: cholinergic functional compensation. Pharmacol Biochem Behav. 2011;99(2):254-61. doi: 10.1016/j.pbb.2011.02.022.

40. Bentley P, Driver J, Dolan RJ. Cholinergic modulation of cognition: insights from human pharmacological functional neuroimaging. Prog Neurobiol. 2011;94(4):360-88. doi: 10.1016/j.pneurobio.2011.06.002.

41. Glick SD, Greenstein S. Differential effects of scopolamine and mecamylamine on passive avoidance behavior. Life Sci I. 1972;11(4):169-79.

42. Parikh V, Cole RD, Patel PJ, Poole RL, Gould TJ. Cognitive control deficits during mecamylamine-precipitated withdrawal in mice: Possible links to frontostriatal BDNF imbalance. Neurobiol Learn Mem. 2016;128:110-6. doi: 10.1016/j. nlm.2016.01.003.

43. Nickell JR, Grinevich VP, Siripurapu KB, Smith AM, Dwoskin LP. Potential therapeutic uses of mecamylamine and its stereoisomers. Pharmacol Biochem Behav. 2013;108:28-43. doi: 10.1016/j.pbb.2013.04.005.

44. Tadavarty R, Kaan TK, Sastry BR. Long-term depression of excitatory synaptic transmission in rat hippocampal CA1 neurons following sleep-deprivation. Exp Neurol.
2009;216(1):239-42. doi: 10.1016/j.expneurol.2008.11.012.

45. Foster TC, Kumar A. Susceptibility to induction of long-term depression is associated with impaired memory in aged Fischer 344 rats. Neurobiol Learn Mem. 2007;87(4):522-35. doi: 10.1016/j.nlm.2006.12.009.

46. Kopp C, Longordo F, Nicholson JR, Luthi A. Insufficient sleep reversibly alters bidirectional synaptic plasticity and NMDA receptor function. J Neurosci. 2006;26(48):12456-65. doi: 10.1523/jneurosci.2702-06.2006.

47. Basheer R, Strecker RE, Thakkar MM, McCarley RW. Adenosine and sleep-wake regulation. Prog Neurobiol. 2004;73(6):37996. doi: 10.1016/j.pneurobio.2004.06.004.

48. Bjorness TE, Greene RW. Adenosine and sleep. Curr Neuropharmacol. 2009;7(3):238-45. doi: $10.2174 / 157015909789152182$.

49. Porkka-Heiskanen T, Strecker RE, Thakkar M, Bjorkum AA, Greene RW, McCarley RW. Adenosine: a mediator of the sleep-inducing effects of prolonged wakefulness. Science. 1997;276(5316):1265-8.

50. Schmitt LI, Sims RE, Dale N, Haydon PG. Wakefulness affects synaptic and network activity by increasing extracellular astrocyte-derived adenosine. J Neurosci. 2012;32(13):4417-25. doi: 10.1523/jneurosci.5689-11.2012.

51. Florian C, Vecsey CG, Halassa MM, Haydon PG, Abel T. Astrocyte-derived adenosine and $\mathrm{A} 1$ receptor activity contribute to sleep loss-induced deficits in hippocampal synaptic plasticity and memory in mice. J Neurosci. 2011;31(19):6956-62. doi: 10.1523/jneurosci.5761-10.2011.

52. Huang EJ, Reichardt LF. Neurotrophins: roles in neuronal development and function. Annu Rev Neurosci. 2001;24:677736. doi: 10.1146/annurev.neuro.24.1.677.

53. Park H, Poo MM. Neurotrophin regulation of neural circuit development and function. Nat Rev Neurosci. 2013;14(1):723. doi: 10.1038/nrn3379.

54. Hernandez PJ, Abel T. A molecular basis for interactions between sleep and memory. Sleep Med Clin. 2011;6(1):71-84. doi: 10.1016/j.jsmc.2010.12.004.

55. Cao X, Cui Z, Feng R, Tang YP, Qin Z, Mei B, et al. Maintenance of superior learning and memory function in NR2B transgenic mice during ageing. Eur J Neurosci. 2007;25(6):1815-22. doi: 10.1111/j.1460-9568.2007.05431.x.

56. Morris RG, Anderson E, Lynch GS, Baudry M. Selective impairment of learning and blockade of long-term potentiation by an N-methyl-D-aspartate receptor antagonist, AP5. Nature. 1986;319(6056):774-6. doi: 10.1038/319774a0.

57. Cortese BM, Mitchell TR, Galloway MP, Prevost KE, Fang J, Moore GJ, et al. Region-specific alteration in brain glutamate: possible relationship to risk-taking behavior. Physiol Behav. 2010;99(4):445-50. doi: 10.1016/j.physbeh.2009.12.005.

58. Dash MB, Douglas CL, Vyazovskiy VV, Cirelli C, Tononi G. Long-term homeostasis of extracellular glutamate in the rat cerebral cortex across sleep and waking states. J Neurosci. 2009;29(3):620-9. doi: 10.1523/jneurosci.5486-08.2009.

59. Kruk-Slomka M, Budzynska B, Biala G. Involvement of cholinergic receptors in the different stages of memory measured in the modified elevated plus maze test in mice. Pharmacol Rep. 2012;64(5):1066-80.

60. Beer AL, Vartak D, Greenlee MW. Nicotine facilitates memory consolidation in perceptual learning. Neuropharmacology. 2013;64:443-51. doi: 10.1016/j.neuropharm.2012.06.019.

61. Shen JX, Yakel JL. Nicotinic acetylcholine receptor-mediated calcium signaling in the nervous system. Acta Pharmacol Sin. 2009;30(6):673-80. doi: 10.1038/aps.2009.64.

62. Fu H, Dou J, Li W, Luo J, Li KC, Lam CS, et al. Mecamylamine prevents neuronal apoptosis induced by glutamate and low potassium via differential anticholinergic-independent mechanisms. Neuropharmacology. 2008;54(4):755-65. doi: 10.1016/j.neuropharm.2007.12.003. 